\title{
Multiscaling analysis of high resolution space-time lidar-rainfall
}

\author{
P. V. Mandapaka, P. Lewandowski, W. E. Eichinger, and W. F. Krajewski \\ IIHR-Hydroscience \& Engineering, The University of Iowa, Iowa City, Iowa, USA \\ Received: 5 April 2009 - Revised: 27 August 2009 - Accepted: 2 September 2009 - Published: 24 September 2009
}

\begin{abstract}
In this study, we report results from scaling analysis of $2.5 \mathrm{~m}$ spatial and $1 \mathrm{~s}$ temporal resolution lidar-rainfall data. The high resolution spatial and temporal data from the same observing system allows us to investigate the variability of rainfall at very small scales ranging from few meters to $\sim 1 \mathrm{~km}$ in space and few seconds to $\sim 30 \mathrm{~min}$ in time. The results suggest multiscaling behaviour in the lidar-rainfall with the scaling regime extending down to the resolution of the data. The results also indicate the existence of a space-time transformation of the form $t \sim L^{z}$ at very small scales, where $t$ is the time lag, $L$ is the spatial averaging scale and $z$ is the dynamic scaling exponent.
\end{abstract}

\section{Introduction}

Spatial and temporal variability of rainfall across multiple scales is of fundamental interest to meteorologists and hydrologists. In the last two decades, multiscaling-based framework has been increasingly used by researchers to statistically characterize the rainfall variability over a range of temporal and spatial scales (e.g., Schertzer and Lovejoy, 1987; Tessier et al., 1993; Gupta and Waymire, 1993; Georgakakos et al., 1994; Veneziano et al., 1996; Venugopal et al., 1999; Lilley et al., 2006; Lovejoy and Schertzer, 2006; Lovejoy et al., 2008). While the temporal scales varied from few seconds to years (e.g., Georgakakos et al., 1994; Olsson et al., 1993), the spatial scales varied from few hundreds of meters to continental scales (e.g., Lovejoy and Schertzer, 2006; Lovejoy et al., 2008). Although rainfall is comprised of individual rain drops, it is usually studied as a continuous field under the assumption of large number $(N)$ of drops. Lilley et al. (2006) showed evidence of multifractal nature of this N-limit in rain based on the data collected during the HYDROP experiment (Desaulniers-Soucy et al., 2001). In

Correspondence to: P. V. Mandapaka (pmandapa@engineering.uiowa.edu) this study we aim to investigate the presence of multiscaling in rainfall at very small scales ranging from few meters to $\sim 1 \mathrm{~km}$ in space and few seconds to $\sim 30 \mathrm{~min}$ in time using the $2.5 \mathrm{~m}$ and $1 \mathrm{~s}$ resolution rainfall data measured by lidar over Iowa City, Iowa, USA (Lewandowski et al., 2009). Our study therefore contributes towards understanding the multiscale statistical properties of rainfall at space-time scales that received little attention, as shown in the following brief review of the literature.

A physical process is said to be scale-invariant or scaling, if large scale and small scale structures are related by a scale-changing operation that involves only the scale ratio and an exponent (e.g., Schertzer and Lovejoy, 1987). If different exponents are required to describe the scaling behavior of different moments, then the process is said to be multiscaling. Studies have shown that multiscaling behaviour is a result of multiplicative cascades, where large scale structures feed small scale structures (e.g., Schertzer and Lovejoy, 1987; Lovejoy et al., 1990). This results in higher and higher intensities being concentrated into smaller and smaller areas, which is true of rainfall process. In addition to understanding rainfall processes across multiple scales, other attractive features of the multiscaling framework are that parsimonious models can be developed to (1) generate synthetic rainfall fields at a given resolution, and (2) statistically downscale rainfall fields to a desired resolution.

The first step in the scaling analysis of rainfall is usually to investigate the presence of scale-invariance using the power spectrum analysis. After the power spectrum analysis, tools such as moment scaling, structure functions, and probability distribution multiple scaling (e.g., Lovejoy and Schertzer, 1990; Nykanen and Harris, 2003) are applied to detect the presence of multiscaling behaviour in the rainfall. Since we are interested in rainfall variability at smaller scales, we present here a short overview of the studies that employed high resolution rainfall data (scales below $1 \mathrm{~min}$ in time and $1 \mathrm{~km}$ in space). We follow this review with description of our experimental setup and then describe our analysis and results. 


\subsection{Brief review of temporal analyses}

Scaling analysis of rainfall in time has been most often performed using the rainfall measured by rain gauges. Crane (1990) analyzed the 30-s rain gauge time series of a rain storm in Germany and reported the spectral slopes closer to turbulence spectral slopes of $5 / 3$, and 3 . Olsson et al. (1993) analyzed two years of 1-min resolution rainfall data collected at 12 locations in the city of Lund, Sweden. They reported that the power spectrum is scale-invariant from $40 \mathrm{~min}$ to approximately $5000 \mathrm{~min}$ with an exponent of $5 / 3$. They also reported a break in scaling of power spectrum at $40-50 \mathrm{~min}$, which corresponds to average rainfall event duration. The scale break was also noticed in their box dimension analysis around 20 min to one hour depending on the low rainfall threshold. Georgakakos et al. (1994) analyzed the $5 \mathrm{~s}$ resolution rainfall time series of seven storms that occurred between May 1990 and April 1991. Based on the power spectrum analysis, they reported a scaling regime from $10 \mathrm{~s}$ to $20 \mathrm{~s}$ for all the storms except one storm for which they reported a regime from $20 \mathrm{~s}$ to $10 \mathrm{~min}$. They have also suggested of a possibility that for a considerably larger sample size (longer duration events), the scaling regime might increase. The scaling exponents estimated in that study ranged from 1.2 to 1.4. Fabry (1996) analyzed data measured by a sonic gauge, rain gauge and vertically pointed radar to determine the scale regimes for the precipitation fields. The sonic gauge had a resolution of $0.1 \mathrm{~s}$, whereas the rain gauge data was at daily scale. The spatial data from the radar was converted to equivalent time data using an echo speed of $25 \mathrm{~m} / \mathrm{s}$. From the power spectra of the sonic gauge data and converted radar data, he reported a scaling range extending from approximately an hour to a few seconds with a scale break and near white noise like behaviour in the spectrum after that. The slope of the power spectrum in this regime was estimated to be 1.40. He further argued inertia and fall speeds of the hydrometeors resulted in the scale break in the precipitation field and the white noise spectrum at higher frequencies.

Harris et al. (1996) investigated the orographic influence on the multiscaling properties of the 15-s resolution rainfall time series collected by rain gauges during June 1993, May 1994, and November 1994 in Southern Alps, New Zealand. They reported that the power spectrum scaling regime and the exponent depended on the elevation of the rain gauge. The scaling regime was longer and the exponent decreased (from $\sim 1.5$ to $\sim 0.9$ ) as one moved to higher elevations. From the moment scaling analysis, they noticed that the intermittency in the rainfall decreased with the increase in elevation. Veneziano et al. (1996) analyzed the same data as in Georgakakos et al. (1994) and showed that the power spectrum of the logarithm of the rainfall time series has a segmented form with four distinct scaling regimes with exponents of 1.6, 2.7, 0.7, and 1.9 in the order of increasing frequency. However, they argued that the rainfall is not multiscaling and proposed a model that satisfies the conditions of stationarity, non-negativity and multiplicative structure observed in the rainfall records. Menabde et al. (1997b) analyzed $15 \mathrm{~s}$ resolution rainfall data of length $17 \mathrm{~h}$ measured by electronic rain gauges over Norfolk Island and Matawai regions in New Zealand. They reported a scaling behavior in the spectrum from the scales of $17 \mathrm{~h}$ down to approximately $4 \mathrm{~min}$. They did not consider smaller scales to avoid possible instrumentation effects on the spectrum. Nikolopoulos et al. (2008) compared the power spectra of stratiform and convective rainfall measured by two vertically pointing radars (S-band and Xband) at three different heights. The resolution and the length of the rainfall data were $9 \mathrm{~s}$ and 1 day, respectively. The spectra from both the radars displayed scaling behaviour for both the storms with spectral exponents ranging from 1.36 to 3.34. In general, they have reported a scaling regime extending from about $20 \mathrm{~s}$ to approximately an hour. However, the scaling regimes changed with the type of the storm and also with the altitude. Though they noticed a white noise like behaviour in the spectrum at higher frequencies (similar to Fabry, 1996) for the stratiform rainfall, the power spectrum of convective rainfall did not present such behaviour.

\subsection{Brief review of spatial analyses}

The spatial multiscaling analyses were usually carried out with the data provided by ground-based and space-based remote sensing platforms, although there were few studies which converted temporal rainfall data from rain gauges into spatial data from Taylor's hypothesis. In this section, we only present a short review of studies that used high resolution (less than or equal to $1 \mathrm{~km}$ ) spatial data.

Tessier et al. (1993) analyzed the scaling properties of horizontal cloud radiances obtained from satellites in different frequency bands, and horizontal and vertical radar reflectivity fields. From the power spectrum of the fields, they concluded that the horizontal cloud radiances scale over a wide range of approximately $200 \mathrm{~m}$ to $2000 \mathrm{~km}$ and horizontal radar reflectivity fields display scaling from approximately $75 \mathrm{~m}$ to $10 \mathrm{~km}$. For the vertical radar reflectivity fields, the one-dimensional spatial power spectrum averaged in time displayed scaling down to $100 \mathrm{~m}$. Menabde et al. (1997a) analyzed 50 two-dimensional rainfall fields obtained from a mobile X-band radar in New Zealand at a resolution of $120 \mathrm{~min}$. The individual as well as ensemble averaged power spectra displayed a good scaling regime from $30 \mathrm{~km}$ down to $240 \mathrm{~m}$. The scaling exponent of ensemble averaged spectrum was estimated to be 2.38. Nykanen and Harris (2003) analyzed the orographic effects on multiscaling characteristics of three storms in the Blue Ridge Mountains of Virginia. The power spectrum exponents for the three storms respectively were $2.29,2.58$, and 2.69 . Though the upper limit of the scaling regime varied from storm to storm, they reported that all the spectra were scaling down to $1 \mathrm{~km}$. They have reported a decreasing trend of scaling range of power spectrum with increasing topographic elevation. However, they have 
also indicated that the relation of multiscaling characteristics with rainfall is not universal and depends on the surrounding meteorology.

\subsection{Brief review of spatio-temporal analyses}

The studies discussed so far have analyzed the spatial and temporal variability of rainfall independently. However, some studies have performed simultaneous multiscaling analysis of spatially variable rainfall field that evolves over time (e.g., Marsan et al., 1996; Venugopal et al., 1999). Marsan et al. (1996) proposed a space-time rainfall model based on three-dimensional anisotropic multiplicative cascades taking into account scaling anisotropy between space and time characterized by exponent $H$ and causality. The value of $H$ was found to be -0.11 and -0.09 for $\mathrm{x}-\mathrm{t}$ and $\mathrm{y}-\mathrm{t}$ sections of NEXRAD radar-rainfall datasets of $8-\mathrm{km}$ resolution in space and 15-min resolution in time. Based on the analysis of convective storms in Darwin, Australia, Venugopal et al. (1999) reported an existence of a power-law space-time transformation of the form $t \sim L^{z}$ ( $t$ is the time lag, and $L$ is the spatial averaging scale) such that rainfall evolution remains invariant over a range of scales $(2 \mathrm{~km}$ to $20 \mathrm{~km}$ in space and $10 \mathrm{~min}$ to several hours in time). The exponent $z$ called the Dynamic scaling exponent was found to vary between 0.6 and 1.2. In this study we employ the methodology proposed by Venugopal et al. (1999) to investigate the presence of power-law space-time transformation.

From the above discussion it is clear that the scaling exponents and the regimes not only depend on natural causes such as orographic forcing and type of rainfall but also on artificial aspects such as the type of the measuring device, and the resolution of the data. According to Tessier et al. (1993), if rainfall is related to atmospheric turbulence, any break in rainfall scaling could be due to a break in the scaling of atmospheric turbulence. While some studies confirmed that the power spectrum scales up to the resolution of the instrument, there were some studies which reported that beyond a certain scale, the small scale structure of the rainfall is lost resulting in the white noise like spectrum. From the brief literature review, it is also clear that the highest temporal resolution of the rainfall analyzed was about $0.1 \mathrm{~s}$ (Fabry, 1996) and the highest spatial resolution was approximately $75 \mathrm{~m}$ (Tessier et al., 1993).

Our main intention behind this study is to introduce the unique high-resolution space-time lidar-rainfall dataset, perform preliminary multiscaling analysis at the range of scales rarely (if ever) analyzed. The results from this study would therefore help us better understand the scaling properties of rainfall at very small scales. The paper is organized as follows. Following the Introduction, Sect. 2 describes the data and Sect. 3 gives a short description of the multiscaling analysis tools employed in this study. The results are discussed in the Sect. 4 followed by the summary and conclusions in Sect. 5.

\section{Data}

Lidar (Light Detection and Ranging) is an active remote sensing instrument primarily used in atmospheric applications (planetary boundary layer, aerosols, water vapour studies, etc.). Its design is based on the same principles as radars with the exception that lidar uses a laser as its source of electromagnetic radiation. After emitting a very short pulse of laser light $(\sim 10 \mathrm{~ns})$, lidar measures the amount of light returning to its detector as a function of time (or distance because the speed of light is fixed). A single lidar profile contains information about the amount of energy that was scattered from the pulse by hydrometeors at various ranges along the path. Lidar does not provide a direct measurement of rainfall intensity. A complicated data processing is required in order to invert the raw lidar data to rain intensities. The lidar data processing involves two major steps of inversion: (i) determination of extinction coefficients from the raw lidar data and (ii) calculating corresponding rain rates.

The lidar data used in this scaling study was originally taken on 20 September 2002 in Iowa City, IA. The lidar system was equipped with a 1.064 micron Nd:YAG laser, attached to a Cassagrain telescope with a primary mirror window of $25 \mathrm{~cm}$ and focal length of $2.5 \mathrm{~m}$. The laser pulsed at $50 \mathrm{~Hz}$ with $25 \mathrm{~mJ}$ of energy per pulse. The signal on the detector was digitized and consecutive profiles were averaged, resulting in $2.5 \mathrm{~m}$ spatial and $1 \mathrm{~s}$ temporal resolution. The length of the dataset used in this study is approximately $75 \mathrm{~min}$ and the maximum distance for which the data is available at each time step is approximately $3 \mathrm{~km}$. To avoid any corrupted data due to the lidar signal attenuation by the heavy rainfall, we only considered the data up to a distance at which the rainfall rate was less than $50 \mathrm{~mm} / \mathrm{h}$. We also did not include the first $150 \mathrm{~m}$ of data due to the instrumentation issues. Therefore, the spatial range varied at each time step with the shortest distance range of $760 \mathrm{~m}$ and longest range of approximately $2350 \mathrm{~m}$. A detailed description of the lidar data processing performed on the lidar data for this study is presented in Lewandowski et al. (2009).

\section{Analysis tools}

\subsection{Power spectrum analysis}

We have seen in the Sect. 1 that the Fourier power spectrum was one of the most widely used tools to detect the presence of scale-invariance in rainfall. A power spectrum that displays log-log linearity within a certain range of frequencies can be represented by a simple power law of the form:

$E(f)=f^{-\beta}$

where $f$ is the frequency and exponent is the slope $\beta$. The process is then scale-invariant in nature. The power spectrum should display log-log linearity as represented by Eq. (1) for 
a range of scales. However, there is no definite rule that gives the minimum range for which the spectrum should be log-log linear. The log-log linearity can be checked by performing local linear analysis and also based on the $R^{2}$ value in linear regression. The corresponding scaling regime should be at least of such a length that there are negligible sampling effects in the regression analysis. Steeper the power spectrum smoother and more organized the rainfall field, as in the case of convective storms (e.g., Nykanen and Harris, 2003).

\subsection{Moment scaling analysis}

The next tool we used in this study is the moment scaling analysis to investigate the presence of multifractality. For a multifractal process, it has been shown (e.g., Menabde et al., 1997a) that the spectral slope $\beta$ is always less than dimension $\mathrm{D}$ of the field. If $\beta>\mathrm{D}$, which is often the case with geophysical phenomena including rainfall, moment scaling analysis is performed on their fluctuations. These can be obtained either by fractional differentiation (e.g., Schertzer and Lovejoy, 1987; Nykanen and Harris, 2003) or by taking small-scale fluctuations of the original field (e.g., Tessier et al., 1993; Menabde et al., 1997a). In this study, we adopted the latter approach.

In the spatial moment scaling analysis, we obtained the rainfall fluctuations in space $\varphi\left(x_{i}, t_{j}\right)$ at each time instant $t_{j}$ as

$\varphi\left(x_{i}, t_{j}\right)=\left|R\left(x_{i+1}, t_{j}\right)-R\left(x_{i}, t_{j}\right)\right|$

where $R\left(x_{i}, t_{j}\right)$ is the lidar-rainfall value at pixel $x_{i}$ for time instant $t_{j}$. The fluctuations were averaged over a range of spatial scales $L$ (Scale Ratio $\lambda=$ Total Spatial Distance $L$ ) to obtain $\varphi_{\lambda}\left(x, t_{j}\right)$. The $\varphi_{\lambda}\left(x, t_{j}\right)$ values for all time steps were pooled together and the statistical moments $M_{q}(\lambda)$ of various moment orders $q$ were estimated for each scale ratio $\lambda$.

$M_{q}(\lambda)=\left\langle\varphi_{\lambda}\left(x, t_{j}\right)^{q}\right\rangle$ for all $t_{j}$

where, $\langle\cdot\rangle$ denotes the ensemble average. The procedure for the temporal moment scaling analysis was similar to that of spatial analysis. Here, we fixed the spatial location and obtained rainfall fluctuations in time. The fluctuations were then averaged over a range of temporal scales and the statistical moments of various orders were estimated.

The rainfall fluctuations are said to be multifractal if there exists a scaling relationship of the form:

$M_{q}(\lambda) \sim \lambda^{K(q)}$

and if $K(q)$ (slope of $M_{q}(\lambda)$ versus $\lambda$ in the logarithmic domain) is a nonlinear function of $q$. The nonlinear function is called moment scaling function or simply $K(q)$ function and the exponent $K(q)$ is referred to as moment scaling exponent. Theoretically, $K(q)$ is required for $q$ ranging from 0 to $\infty$ to fully characterize the multifractality in the rainfall fluctuation fields. However, Tessier et al. (1993) proposed a universal multifractal model for the $K(q)$ based on multiplicative cascades consisting of parameters $\alpha$, and $C_{1}$.

$K(q)=\frac{C_{1}}{\alpha-1}\left(q^{\alpha}-q\right) 0 \leq \alpha<1$ and $1<\alpha \leq 2$

$K(q)=C_{1} q \log q \quad \alpha=1$

The parameter $\alpha$ is the Levy-stable (or multifractality) index and indicates the probability distribution from which the weights are generated in the cascading process. The case $0<\alpha<2(\alpha \neq 1)$ corresponds to $\log ($ Levy) multifractals, and if $\alpha=1$ the multifractal process is $\log$ (Cauchy) (e.g., Tessier et al., 1993). The case with $\alpha=2$ corresponds to lognormal multifractals. $C_{1}$ is the intermittency parameter that characterizes the sparseness of the mean.

In this study, the parameters $C_{1}$ and $\alpha$ were obtained using the double trace moments (DTM) technique (e.g., Tessier et al., 1993). In the DTM technique, we take various powers $\eta$ of the rainfall fluctuations at their highest resolution, then average the powered fluctuations to various spatial and temporal scales (with scale ratio $\lambda$ ), and estimate the statistical moments of various orders $q$.

$M_{\eta, q}(\lambda)=\left\langle\left[\varphi^{\eta}\left(x, t_{j}\right)\right]_{\lambda}^{q}\right\rangle$ for all $t_{j}$

In case of universality, the statistical moments will depend on scale ratio as:

$M_{\eta, q}(\lambda) \sim \lambda^{K(q, \eta)-(q-1) \mathrm{D}}$

and

$K(q, \eta)=\eta^{\alpha} \cdot K(q)$

Therefore $\alpha$ is the slope of $K(q, \eta)$ versus $\eta$ in a double logarithmic plot for a fixed $q$. The value of $C_{1}$ can be obtained by plugging $\alpha$ in Eq. (5) for a fixed $q$. For a detailed description of the DTM technique, the reader is referred to Tessier et al. (1993).

\subsection{Dynamic scaling analysis}

So far we have analyzed spatial and temporal components of rainfall independent of each other. Since rainfall is a timeevolving process, the spatial and temporal variability are not independent of each other. Simultaneous multifractal analyses allow studying the temporal evolution of rainfall at multiple spatial scales. In this study we adopted the framework proposed by Venugopal et al. (1999), which consists of estimating the probability density function (PDF) of the following statistic:

$\Delta \ln I_{i, \tau}(L, t)=\ln I_{i}^{L}(\tau+t)-\ln I_{i}^{L}(\tau)$

where $I_{i}^{L}(\tau)$ is the nonzero rainfall intensity at location $i$, time instant $\tau$, for spatial scale $L$, and $t$ denotes the time lag over which the rainfall evolution is measured. Venugopal et al. (1999) chose the above statistic based on the evidence 
that the relative changes in the spatial and temporal rainfall fluctuations were independent of the intensities (e.g., Perica and Foufoula-Georgiou, 1996; Venugopal et al., 1999). $\Delta \ln I_{i, \tau}(L, t)$ was estimated in the following manner.

At each instant in time $\tau$, the rainfall intensities $I_{i}^{L}(\tau)$ were obtained for spatial scales $L$ varying from spatial resolution of the data to the scale allowed by the distance range. For example, $L$ varies as $2.5 \mathrm{~m}, 5 \mathrm{~m}, 10 \mathrm{~m}$ etc. for a data with spatial resolution of $2.5 \mathrm{~m} . \Delta \ln I_{i, \tau}(L, t)$ was then estimated using Eq. (9), where $t$ varied from the temporal resolution of the data to the lag allowed by the length of the dataset. The $\Delta \ln I_{i, \tau}(L, t)$ values can be pooled and the PDFs can be obtained only when their statistical properties do not depend significantly on the absolute time coordinate $\tau$. The standard deviation $\sigma\left(\Delta \ln I_{i, \tau}(L, t)\right)$ was estimated at each time instant to identify such stationary region. Within the stationary region, the standard deviation $\Sigma(L, t)$ of the PDFs of $\Delta \ln I_{i, \tau}(L, t)$ was estimated for each combination of $L$ and $t$. Then the pairs of $L$ and $t$ are found for which $\Sigma(L, t)$ is constant. If the values of $L$ and $t$ display log-log linearity, then there exists a space-time transformation of the form $t \sim L^{z}$ across a range of scales. For a much detailed description of the algorithm, the reader is referred to Venugopal et al. (1999).

\section{Results and discussion}

\subsection{Power spectrum}

\subsubsection{Temporal spectrum}

At each point along the distance axis, Fast Fourier Transform algorithm is applied on the time series of the lidar data to obtain the temporal power spectrum. The average temporal power spectrum was estimated by averaging the individual power spectra for the time series corresponding to spatial locations from $150 \mathrm{~m}$ to $910 \mathrm{~m}$. The range was selected such that we have continuous time series of lidar-rainfall data. Further, only those individual spectra that display scaling behavior with $R^{2}>0.95$ was used to estimate the average spectrum (Fig. 1a).

We carried out the local linear analysis to check the log$\log$ linearity of the average power spectrum and the departure from scaling behavior. A window, whose size varied logarithmically, was moved along the frequency axis of the temporal power spectrum and linear regression was performed within that particular window. Logarithmic sized window was selected so that the window size is uniform in a log-log plot. The slope varied from -0.50 to -2.0 with a sharp transition between frequencies corresponding to $80 \mathrm{~s}$ and $140 \mathrm{~s}$. From $2 \mathrm{~s}$ up to $80 \mathrm{~s}$, the slope varied from -1.75 to -2.0 with a coefficient of variation $(\mathrm{CV})$ of 0.037 compared to the full regime, where the $\mathrm{CV}$ was equal to 0.093 . Therefore, we can say that the spectrum is $\log -\log$ linear in the regime $2 \mathrm{~s}$ to $80 \mathrm{~s}$
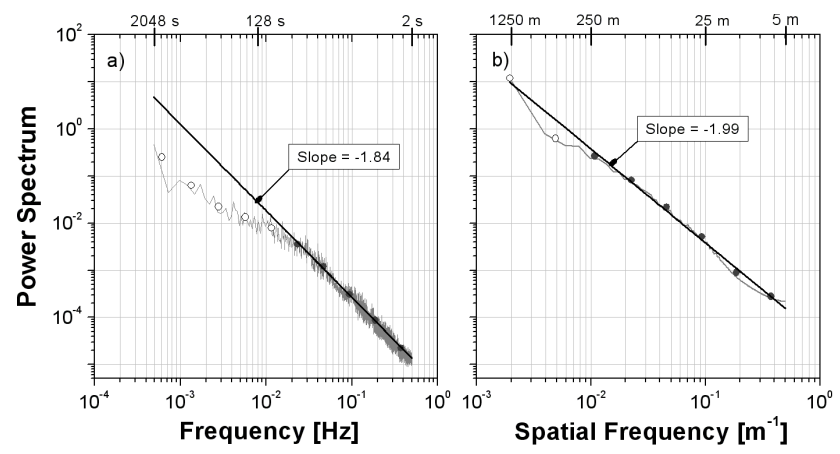

Fig. 1. Temporal and spatial power spectrum of the lidar-rainfall data. The dots indicate the power spectrum averaged in logarithmic (octave) bins. The slope of the regression line fitted to the octavebinned spectrum is also indicated on each panel.

with a departure from scaling around the region $80 \mathrm{~s}$ to $140 \mathrm{~s}$ (Fig. 1a). The departure could be due to the limited sample of the data resulting in sampling issues towards lower frequencies. A thorough analysis with a large dataset is required to check if the scale break (if exists) is related to characteristic time scale in rainfall.

The average spectrum was then octave-binned (e.g., Harris et al., 1996) in this region to avoid excessive weighting on higher frequencies, and the regression analysis was carried out on the octave-binned spectrum. Figure 1a shows the octave-binned spectrum included in the regression analysis (solid gray circles) along with the fitted regression line. The slope of the regression line fitted to the octave-binned average power spectrum was -1.84 with an $R^{2}$ value of 0.99 (Fig. 1a). Further, the average of the slopes of individual power spectra was equal to -1.81 . From these values, it can be said that the average power spectrum is indeed representative of the individual spectra. We refrained from performing linear regression for lower frequencies (empty circles in Fig. 1a) as they are dominated by the sampling effects. Unlike Fabry (1996) and Nikolopoulos et al. (2008), we did not notice any white noise like spectrum at high frequencies. That is, scale-invariance extended up to the scales allowed by the resolution of the data.

\subsubsection{Spatial spectrum}

First, we estimated the individual spatial power spectrum at each instant in time with a fixed spatial range of $1250 \mathrm{~m}$. Due to the maximum rainfall threshold of $50 \mathrm{~mm} / \mathrm{h}$, sometimes the available range was found to be less than $1250 \mathrm{~m}$. In such cases, we zero-padded the lidar signal and estimated the individual power spectrum. The individual spectra estimated at each time instant were then averaged to obtain the average spatial spectrum. Further, only those individual spectra that display scaling behavior with $R^{2}>0.95$ was used to estimate the average spectrum. We did not carry out local linear analysis for the spatial spectrum as there were only 
256 points most of which were concentrated towards higher frequencies. The average spectrum was octave-binned and the slope was estimated leaving first two points (Fig. 1b). The first two points were not included to avoid sampling effects. The average value of the slopes of individual spatial power spectra was equal to -2.02 and the slope of average spatial power spectrum was -1.99 with an $R^{2}$ value of 0.99 . From the high value of $R^{2}$, it can be said that the spectrum is $\log$ - $\log$ linear in the regime $5 \mathrm{~m}$ to $250 \mathrm{~m}$. Figure $1 \mathrm{~b}$ shows the average spatial power spectrum, the octave binned average spectrum and the fitted regression line. It is not possible to compare the spatial power spectrum from this study with the ones reported in the literature as most of them were radially averaged two-dimensional power spectra obtained from two-dimensional rainfall fields.

\subsection{Moment scaling}

From the Sect. 4.1, we have seen that the power spectral exponents were greater than the dimension of the data, which is one. Therefore, moment scaling analysis was performed on the absolute fluctuations computed using Eq. (2). In the Fig. 2, we show the statistical moments obtained using Eq. (3) for both time and space. It can be noticed that the moments display log-log linearity with respect to the scale. In time, the scale-invariance was found to hold from the lowest resolution of $1 \mathrm{~s}$ to $512 \mathrm{~s}$ in time and in space it extended from $2.5 \mathrm{~m}$ to $320 \mathrm{~m}$. The scaling regimes in time and space were shorter than those noticed in power spectral analysis in the previous section. The power spectrum and the moment scaling analysis are two different tools to investigate the presence of scaling. While the power spectrum is a secondorder analysis, the moment scaling invlolves estimation of the moments of order ranging from 0.1 to 3.5. In addition, the sampling issues impact these two techniques in a different manner. Therefore, the scaling regimes detected by these tools need not be exactly same.

We did not perform the moving window analysis (like in power spectrum) to check the log-log linearity and the deviation in scaling behavior as we only had 10 points. The $R^{2}$ value for the linear regression in the log-log plot for various moment orders (Fig. 2) was always greater than 0.98. From the high values of $R^{2}$, it can be said that the moments are indeed $\log$ - $\log$ linear. Figure 2 also shows the ordinary least squares regression fit to the statistical moments. The slopes $K(q)$ of the fitted regression lines are plotted against the corresponding moment orders for the temporal and spatial domains in the Fig. 3. For both cases, the slopes vary nonlinearly with the moment order confirming that the rainfall fluctuations are multfractal. Figure 3 also shows the DTM fit and the corresponding universal multifractal parameters for space and time domain. While the intermittency parameter $C_{1}$ was the same for temporal and spatial data, the multifractal parameter $\alpha$ was greater for spatial data (Fig. 3).

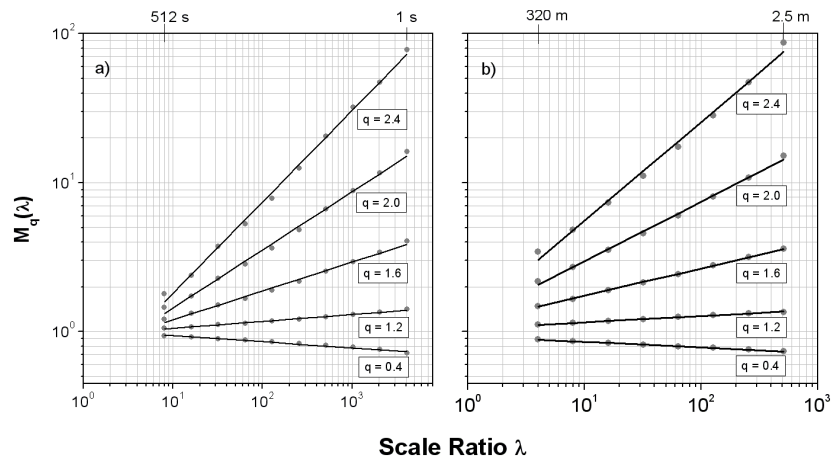

Fig. 2. Variation of (a) temporal and (b) spatial moments of rainfall fluctuations of various moment orders $q$ with respect to scale ratio $\lambda$. Figure also shows the fitted ordinary linear regression lines.

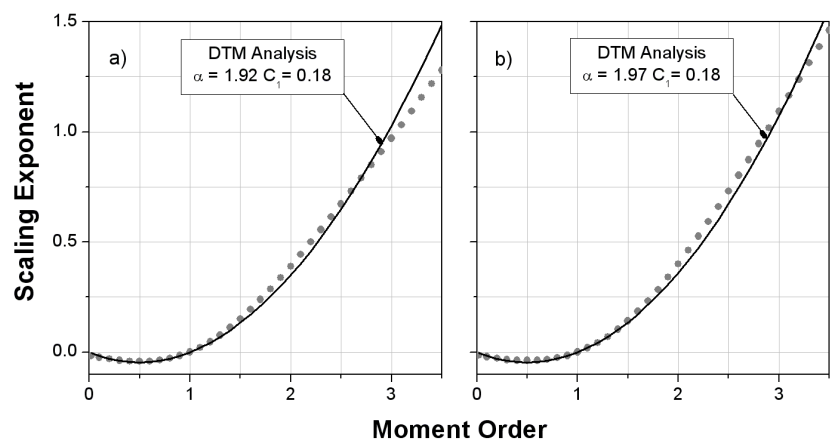

Fig. 3. Variation of (a) temporal and (b) spatial moment scaling exponents with respect to the moment order. The parameters of the universal multifractal model are given in the inset.

\subsection{Dynamic scaling}

As mentioned in Sect. 3.3, dynamic scaling analysis has to be performed in the region where the statistical properties of the quantity estimated using Eq. (10) do not depend significantly on the absolute time coordinate $\tau$. To identify such stationary region, we moved a window of size $900 \mathrm{~s}$ ( $15 \mathrm{~min})$ along the standard deviation series $\sigma\left(\Delta \ln I_{i, t}(8,8)\right)$ and estimated the $\mathrm{CV}$. The window that resulted in the least $\mathrm{CV}$ was selected for the dynamic scaling analysis. The coefficient of variation for the corresponding time period is 0.39 . The overall standard deviation $\Sigma(L, t)$ was then estimated for the stationary region for each combination of $L$ and $t$. In the Fig. 4, we plot the pairs of $L$ and $t$ for which the standard deviation $\Sigma$ was constant. From the Fig. 4, it can be seen that the relationship between $L$ and $t$ is $\log$ - log linear confirming the presence of dynamic scaling of the form $t \sim L^{z}$ in the lidar-rainfall data.

Due to the restriction imposed by the attenuation of lidar signal by heavy rainfall, the spatial range at each time step varied from approximately $750 \mathrm{~m}$ to $2350 \mathrm{~m}$. The stationarity conditions further limited the length of the dataset to $15 \mathrm{~min}$. Therefore, we have only four to five points as an 


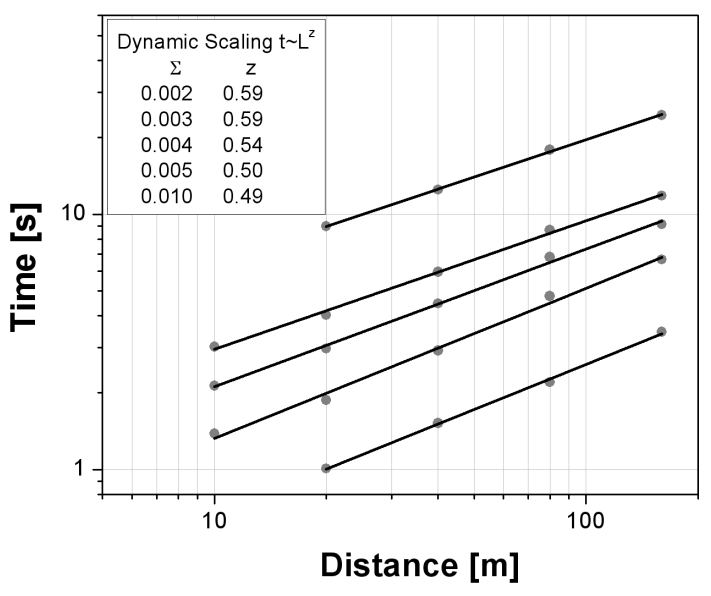

Fig. 4. Evidence of dynamic scaling in the standard deviation of $\Delta \ln I_{i, t}(L, t)$ (Eq. 9). The values of the standard deviations and the corresponding dynamic scaling exponents were given in the inset.

evidence of dynamic scaling in lidar-rainfall (Fig. 4). The scaling range extended from $10 \mathrm{~m}$ to $160 \mathrm{~m}$ in space and from $1 \mathrm{~s}$ to $\sim 20 \mathrm{~s}$ in time. However, it should be noted that for a dataset with wider spatial range and longer stationary period, one might notice longer scaling regimes. The dynamic scaling exponent $z$ is also shown in the Fig. 4 along with the corresponding standard deviations. The value of $z$ varied from 0.49 to 0.59 depending on the standard deviation. The exponents were different compared to Venugopal et al. (1999) who reported exponents in the range of 0.6 to 0.7 for tropical convective storms of Darwin, Australia. The scaling regimes from this study cannot be compared with that of Venugopal et al. (1999) as they analyzed radar-rainfall images with a resolution of $2 \mathrm{~km}$ in space and $10 \mathrm{~min}$ in time.

\section{Summary and conclusions}

We investigated the space-time lidar-rainfall data with a resolution of $2.5 \mathrm{~m}$ in space and $1 \mathrm{~s}$ in time for the presence of scale invariance. The power spectrum analysis suggested that the lidar-rainfall data were scale-invariant with exponents of -1.84 and -1.99 in time and space respectively. The scaling regime in time extended from $2 \mathrm{~s}$ up to $80 \mathrm{~s}$. We noticed a transition region between $80 \mathrm{~s}$ and $140 \mathrm{~s}$, where the power spectrum departs from the scaling behaviour. The scaling regime in space extended from $5 \mathrm{~m}$ to $250 \mathrm{~m}$. We did not observe white noise spectrum at higher frequencies that were reported by some previous studies. In the moment scaling analysis, the statistical moments of the rainfall fluctuations displayed scaling behaviour with scaling regimes extending from $1 \mathrm{~s}$ to $512 \mathrm{~s}$ in time and $2.5 \mathrm{~m}$ to $320 \mathrm{~m}$ in space. The nonlinearity of the moment scaling function indicated that the lidar-rainfall data displayed multiscaling behaviour both in space and time. The $K(q)$ function was then paramete- rized according to the double trace moment technique to obtain the universal multifractal model parameters. The intermittency parameter $C_{1}$ was same for both spatial and temporal lidar-rainfall series. The multifractal index $\alpha$ was equal to 1.92 for temporal and 1.97 for spatial data. The values of $\alpha$ indicate that the lidar-rainfall data corresponds to the $\log$ (Levy) multifractals. We also noticed a space-time transformation of the form $t \sim L^{z}$ in the lidar-rainfall data with an exponent in the range of 0.49 to 0.59 . However, it should be noted that this transformation was observed only for the very small scales in the range of $1 \mathrm{~s}$ to $\sim 20 \mathrm{~s}$ in time and from $10 \mathrm{~m}$ to $160 \mathrm{~m}$ in space.

Our high-resolution dataset allowed us to perform multiscaling analysis at very small scales that received little attention in the literature. However, to really bridge the scale gap, we need to perform an expensive experiment with lidars, rain gauges, radars and satellites observing the same rainfall system for a considerable time period. Such an experiment should be designed so that there is some overlap in the scales observed by different instruments. Besides bridging the scale gap, such an experiment will also provide insights into the physical mechanisms responsible for the observed scaling behavior in rainfall datasets.

Acknowledgements. The authors acknowledge useful discussions with Deborah Nykanen and V. Venugopal.

Edited by: J. Kurths

Reviewed by: two anonymous referees

\section{References}

Crane, R. K.: Space-time structure of rain rate fields, J. Geophys. Res., 95, 2011-2020, 1990.

Desaulniers-Soucy, N., Lovejoy, S., and Schertzer, D.: HYDROP experiment: an empirical method for the determination of the continuum limit in rain, Atmos. Res., 59-60, 163-197, 2001.

Fabry, F.: On the determination of scale ranges for precipitation fields, J. Geophys. Res., 101, 12819-12826, 1996.

Georgakakos, K. P., Carsteanu, A. A., Sturdevant, P. L., and Cramer, J. A.: Observation and analysis of Midwestern rain rates, J. Appl. Meteorol., 33, 1433-1444, 1994.

Gupta, V. K. and Waymire, E. C.: A statistical analysis of mesoscale rainfall as a random cascade, J. Appl. Meteorol., 32, 251-267, 1993.

Harris, D., Menabde, M., Seed, A., and Austin, G.: Multifractal characterization of rain fields with a strong orographic influence, J. Geophys. Res., 101, 26405-26414, 1996.

Lewandowski, P., Eichinger, W. E., Kruger, A., and Krajewski, W. F.: Lidar-based estimation of small-scale rainfall: empirical evidence, J. Atmos. Ocean. Tech., 26, 656-664, 2009.

Lilley, M., Lovejoy, S., Desaulniers-Soucy, N., and Schertzer, D.: Multifractal large number of drops limit in rain, J. Hydrol., 328, 20-37, 2006.

Lovejoy, S. and Schertzer, D.: Multifractals, universality classes and satellite and radar measurements of cloud and rain fields, J. Geophys. Res., 95, 2021-2034, 1990. 
Lovejoy, S. and Schertzer, D.: Multifractals, cloud radiances and rain, J. Hydrol., 322, 59-88, 2006.

Lovejoy, S., Schertzer, D., and Allaire, V. C.: The remarkable wide range spatial scaling of TRMM precipitation, Atmos. Res., 90, 10-32, 2008.

Marsan, D., Schertzer, D., and Lovejoy, S.: Causal space-time multifractal proceses: Predictability and forecasting of rain fields, J. Geophys. Res., 101, 26333-26346, 1996.

Menabde, M., Seed, A., Harris, D., and Austin, G.: Self-similar random fields and rainfall simulation, J. Geophys. Res., 102, 1350913515, 1997a.

Menabde, M., Harris, D., Seed, A., Austin, G., and Stow, D.: Multiscaling properties of rainfall and bounded random cascades, Water Resour. Res., 33, 2823-2830, 1997 b.

Nikolopoulos, E. I., Kruger, A., Krajewski, W. F., Williams, C. R., and Gage, K. S.: Comparative rainfall data analysis from two vertically pointing radars, an optical disdrometer, and a rain gauge, Nonlin. Processes Geophys., 15, 987-997, 2008, http://www.nonlin-processes-geophys.net/15/987/2008/.

Nykanen, D. and Harris, D.: Orographic influences on the multiscale statistical properties of precipitation, J. Geophys. Res., 108(D8), 8381, doi:10.1029/2001JD001518, 2003.
Olsson, J., Niemczynowicz, J., and Berndtsson, R.: Fractal analysis of high-resolution rainfall time series, J. Geophys. Res., 98, 23265-23274, 1993.

Perica, S. and Foufoula-Georgiou, E.: Model for multiscale disaggregation of spatial rainfall based on coupling meteorological and scaling descriptions, J. Geophys. Res., 101, 26347-26361, 1996.

Schertzer, D. and Lovejoy, S.: Physical modelling and analysis of rain and clouds by anisotropic scaling multiplicative processes, J. Geophys. Res, 92, 9693-9714, 1987.

Tessier, Y., Lovejoy, S., and Schertzer, D.: Universal Multifractals: Theory and observations for rain and clouds, J. Appl. Meteorol., 32, 223-250, 1993.

Veneziano, D., Bras, R. L., and Niemann, J. D.: Nonlinearity and self-similarity of rainfall in time and a stochastic model, J. Geophys. Res., 101, 26371-26392, 1996.

Venugopal, V., Foufoula-Georgiou, E., and Sapozhnikov, V.: Evidence of dynamic scaling in space-time rainfall, J. Geophys. Res., 104, 31599-31610, 1999. 